Juliana Fernandes Filgueiras Meireies ${ }^{1}$

Clara Mockdece Neves ${ }^{1}$

Pedro Henrique Berbert de Carvalho ${ }^{2}$ Maria Elisa Caputo Ferreira ${ }^{3}$

\section{Artigo Original}

Palavras-chave
Imagem corporal
Gravidez
Adulto
Estado nutricional
Cuidado pré-natal
Keywords
Body image
Pregnancy
Adult
Nutritional status
Prenatal care

Correspondênci

Juliana Fernandes Filgueiras Meireles Faculdade de Educacacõo Física e Desportos, Campus Universitário Rua José Lourenco Kelmer, $s / n-S a ̃ o$ Pedro

CEP: $36036-900$ Juiz de Fora (MG), Brasil

Recebido

14/05/2015

Aceito com modificacōes

$24 / 06 / 2015$

\title{
Imagem corporal de gestantes: associação com variáveis sociodemográficas, antropométricas e obstétricas
}

\author{
Body image of pregnant women: association with \\ sociodemographic, anthropometric, and obstetric variables
}

\section{Resumo}

OBJETIVO: Comparar atitudes corporais de gestantes nas categorias do índice de massa corporal, em diferentes períodos e condições de risco gestacional, bem como analisar a relação das variáveis de estudo com as atitudes corporais de gestantes. MÉTODOS: Foram incluídas 386 gestantes de todos os períodos gestacionais, que frequentavam o pré-natal nos setores público e privado de uma cidade do Sudeste do Brasil, entre 18 e 46 anos (média de 29,32士6,04 anos), excluindo-se mulheres com dados incompletos. Os instrumentos utilizados para avaliação foram: "Body Attitudes Questionnaire", "Critério de Classificação Econômica Brasil" e questionário sociodemográfico. Ademais, foram coletados dados antropométricos e obstétricos. Foram realizadas análises estatísticas descritivas, comparativas e correlacionais. RESULTADOS: As atitudes corporais das gestantes foram similares entre todos os trimestres gestacionais $(F=0,39 ; p=0,9)$. Já aquelas negativas aumentaram gradativamente entre os grupos de peso baixo $(108,2 \pm 12,5)$, adequado $(116,2 \pm 16,0)$, sobrepeso $(125,1 \pm 14,3)$ e obesidade $(132,9 \pm 16,4)$, e também entre gestantes com risco habitual $(120,0 \pm 17,1)$ e alto risco gestacional $(124,9 \pm 16,7)$. As variáveis sociodemográficas, econômicas e obstétricas não influenciaram significativamente a variância das atitudes corporais. $\bigcirc$ índice de massa corporal explicou 11 1,3\% da variância das atitudes corporais nas grávidas. CONCLUSÕES: $\bigcirc$ estado nutricional e a condição de risco apresentaram relação com atitudes corporais negativas e, por isso, devem ser avaliadas em gestantes, tendo em vista a saúde materna e infantil.

\section{Abstract}

PURPOSE: To compare body attitudes of pregnant women in various body mass index categories, during different gestational periods and under gestational risk conditions, as well as to analyze the association of the study variables with the body attitudes of pregnant women. METHODS: We included 386 pregnant women in all gestational periods, aged 18 to 46 years (mean 29.32 \pm 6.04 years), who attended prenatal care in the public and private sectors of a city in Southeastern Brazil, excluding women with incomplete data. The instruments for assessment were "Body Attitudes Questionnaire", "Critério de Classificação Econômica Brasil", and a sociodemographic questionnaire. In addition, anthropometric and obstetric data were collected. Descriptive, comparative, and correlational statistical analyses were performed. RESULTS: The body attitudes of pregnant women were similar in all pregnancy trimesters ( $F=0.39 ; p=0.9)$. Negative body attitudes increased gradually among low weight (108.2 \pm 12.5 ), appropriate weight (1 16.2 \pm 16.0 ), overweight (125.1 \pm 14.3$)$, and obese (132.9 \pm 16.4$)$ groups, and among pregnancy women with normal $(120.0 \pm 17.1)$ and high-risk pregnancies (124.9 \pm 16.7). The sociodemographic, economic, and obstetric variables did not influence the variance of body attitudes. The body mass index explained $11.3 \%$ of the variance of body attitudes in pregnant women. CONCLUSIONS: Nutritional status and risk conditions showed an association with negative body image and should therefore be evaluated in pregnant women for a better maternal and child health.

\section{Laboratório de Estudos do Corpo da Universidade Federal de Juiz de Fora - UFJF - Juiz de Fora (MG), Brasil.}

IPrograma de Pós-Graduação (Doutorado) em Psicologia, Universidade Federal de Juiz de Fora - UFJF - Juiz de Fora (MG), Brasil.

${ }_{2}^{2}$ Faculdade de Educação Física e Desportos, Universidade Federal de Juiz de Fora - UFJF - Governo Valadares (MG), Brasil.

${ }^{3}$ Faculdade de Educação Física e Desportos, Universidade Federal de Juiz de Fora - UFJF - Juiz de Fora (MG), Brasil.

Conflito de interesses: não há 
Introdução

O período gestacional pode ser considerado como uma situação ímpar na vida de uma mulher, no qual ocorrem rápidas alterações em um curto período, aproximadamente 40 semanas $^{1,2}$. Esse processo compreende tanto modificações fisiológicas quanto psicológicas, sociais e culturais ${ }^{1,3}$. A maioria destas alterações é parte natural da gravidez e de fundamental importância, pois tem como objetivo proporcionar condições para o adequado crescimento e desenvolvimento fetal, em equilíbrio com o organismo materno ${ }^{1,4}$. Além de repercutir de forma expressiva no dia a dia da gestante, tais mudanças podem ter consequências na imagem corporal da mulher ${ }^{1}$.

Somado a isso, a interação social exerce influência na estrutura da imagem corporal, a qual é entendida como a representação mental do corpo ${ }^{5}$. Atualmente, tem-se o padrão feminino corporal direcionado à magreza ${ }^{5}$. O corpo grávido, ao longo dos nove meses de gestação, naturalmente, não se enquadra neste modelo e move-se para longe de tal ideal, já que há ganho de peso e modificação do formato do corpo $^{1,2}$. Por isso, as mudanças físicas podem ser de difícil aceitação pelas mulheres ${ }^{1}$.

Sendo assim, surge a hipótese de que as rápidas mudanças corporais que ocorrem nesse período, somadas à forte idealização social feminina do corpo magro, podem contribuir para que a gestante desenvolva um descontentamento profundo com o próprio corpo e, consequentemente, altere suas atitudes corporais ${ }^{6}$. A preocupação com essas possíveis associações tem despertado o interesse de pesquisadores internacionais ${ }^{6-10}$.

Entretanto, segundo revisão de literatura realizada por Meireles et al. ${ }^{11}$, apenas uma investigação que avaliou a imagem corporal de grávidas brasileiras foi encontrada. Ademais, o mesmo trabalho apontou achados controversos: enquanto algumas pesquisas apontaram para a melhora dos sentimentos relacionados ao corpo, outras identificaram descontentamento com a forma e o peso corporal ao longo da gestação ${ }^{11}$. Dessa forma, ainda são necessários estudos nacionais que objetivem analisar esse construto em gestantes. Salienta-se ainda que, durante a gestação, uma imagem corporal negativa deve ser considerada, pois pode causar implicações graves para a saúde materna e infantil ${ }^{1,6,10}$.

Nesse sentido, o presente estudo teve como objetivo comparar as atitudes relacionadas à imagem corporal de gestantes em seus diferentes períodos de gestação, nas categorias do índice de massa corporal (IMC) e na presença, ou não, da condição de risco gestacional, bem como analisar a relação de variáveis sociodemográficas, econômicas, antropométricas e obstétricas com atitudes relacionadas ao corpo das gestantes.

\section{Métodos}

Esta pesquisa obteve aprovação pelo Comitê de Ética e Pesquisa em Seres Humanos da Universidade Federal de Juiz de Fora (UFJF), sob o número de inscrição 14406413.0.0000.5147, parecer 337.124.

Trata-se de um estudo quantitativo, transversal, descritivo e correlacional ${ }^{12}$. A pesquisa foi realizada na cidade de Juiz de Fora, em Minas Gerais, de forma que a gestante voluntária estivesse frequentando o pré-natal no município. Foram incluídas na pesquisa gestantes de todos os períodos gestacionais, as quais aceitaram participar voluntariamente da pesquisa e assinaram o termo de consentimento livre e esclarecido. Participantes que apresentaram dados incompletos foram excluídas do estudo.

As voluntárias responderam a um questionário sociodemográfico a fim de acessar dados de: idade, nível de escolaridade, estado civil, número de filhos, relação com o pai da criança, apoio familiar, planejamento ou não da gestação e desejo inicial ou não da provocação de um aborto.

Para identificar o nível socioeconômico das gestantes, utilizou-se o "Critério de Classificação Econômica Brasil" "13, que atribui pontos em função de características domiciliares, e a soma da pontuação pode variar de zero a 46, desde $\mathrm{A} 1$ a E.

Dos prontuários das pacientes, foram coletados dados obstétricos (idade gestacional e condição de risco gestacional). A partir da idade gestacional em semanas, adotou-se o ponto de corte estabelecido pelo Ministério da Saúde ${ }^{4}$, que determina: gestantes até a $12^{\text {a }}$ semana primeiro trimestre; entre a 13 e $25^{a}$ semana - segundo trimestre e a partir da $26^{a}$ semana - terceiro trimestre.

Além disso, os médicos responsáveis forneceram dados antropométricos (massa corporal e estatura) das gestantes. Todos os locais utilizavam balanças e estadiômetros da marca Filizola, os quais haviam sido devidamente aferidos e calibrados. Para a classificação do estado nutricional primário, empregou-se o IMC. Após o cálculo, a gestante foi classificada em quatro grupos (baixo peso, adequado, sobrepeso ou obesidade), de acordo com a semana gestacional, como proposto para essa população ${ }^{4}$.

A fim de avaliar as atitudes corporais das gestantes, aplicou-se o Body Attitudes Questionnaire (BAQ) ${ }^{14}$, que é composto por 44 itens. Tal instrumento de autorrelato é pontuado em escala Likert, com cinco opções de alternativas ("Concordo totalmente" até "Discordo totalmente"). O escore total é mensurado pela soma das respostas, podendo variar de 44 a 220, sendo que quanto maior a pontuação, maiores os sentimentos sobre as seis subescalas: "atração física", "autodepreciação", "gordura total", "saliência do corpo", "percepção da gordura da porção inferior do corpo" e "força e aptidão física". Vale destacar que este instrumento tem sido utilizado em mulheres no período 
pré-, durante e pós-gestação $0^{2,9,11,15-18}$. Para a amostra em questão, o alpha de Cronbach encontrado demonstrou boa consistência interna $(\alpha=0,84)$.

Inicialmente, foi realizado contato com obstetras de consultórios particulares e públicos da cidade de Juiz de Fora (MG). Os objetivos e métodos do estudo foram explanados, bem como solicitou-se uma autorização para aplicar os questionários nas gestantes, em salas de espera dos consultórios. Após o consentimento, foram agendados os melhores horários para a coleta de dados, segundo a agenda de cada médico. Nos dias estabelecidos, as gestantes receberam explicação sobre a pesquisa e assinaram o termo de consentimento livre e esclarecido. Todos os questionários foram preenchidos em um mesmo dia e não houve delimitação de tempo para o preenchimento dos mesmos.

Para as análises estatísticas, utilizou-se o software SPSS 19.0 e, em todos os casos, o nível de significância foi de $\mathrm{p}<0,05$. Para cada variável categórica analisada, optou-se pela descrição em frequências absoluta e relativa. Para os dados numéricos, foi realizada análise de médias e desvios padrão. O alpha de Cronbach verificou a consistência interna do BAQ, considerando valor superior a 0,70 como adequado $^{19}$. Observou-se a distribuição dos dados e a hipótese nula foi confirmada, seguindo-se com testes paramétricos. O teste $t$ de Student foi realizado a fim de comparar as médias do BAQ entre as condições de risco (risco habitual e alto risco). O teste de variância ANOVA one-way foi empregado para comparar as médias do BAQ entre os grupos por períodos gestacionais (primeiro, segundo e terceiro trimestre) e estados nutricionais (baixo peso, adequado, sobrepeso e obesidade). Em caso de significância estatística, seguiu-se com o post hoc de Bonferroni. O teste de correlação de Pearson foi realizado a fim de verificar a associação entre as variáveis do estudo (BAQ, idade, IMC, nível socioeconômico e idade gestacional). Em seguida, conduziu-se a regressão linear forward para averiguar a influência que as variáveis, que estiveram significativamente relacionadas, exerciam sobre as atitudes corporais das gestantes (BAQ). A ordem de entrada dos dados respeitou a magnitude do coeficiente de correlação de Pearson, e aquelas com maior grau de associação com o BAQ foram as primeiras a serem introduzidas no modelo de regressão.

\section{Resultados}

Foram coletados dados de 417 gestantes, as quais frequentavam consultas de pré-natal na cidade de Juiz de Fora (MG). Entretanto, 31 foram excluídas por apresentarem dados incompletos. Portanto, a amostra final desta investigação contou com 386 voluntárias (média de 29,32 $\pm 6,04$ anos), sendo 198 do setor público (média de 28,10 $\pm 6,67$ anos) e 188 do privado (média de 30,61 $\pm 5,01$ anos).

Com relação à caracterização sociodemográfica das gestantes, a maioria tinha uma boa relação com o pai da criança $(93,3 \%)$, apoio familiar $(96,4 \%)$ e não havia considerado o aborto $(94,8 \%)$. Além disso, $54,9 \%$ haviam planejado a gravidez. Observa-se que grande parcela da amostra é casada e estava em sua primeira gestação. Destaca-se ainda que as participantes se enquadravam majoritariamente nas classes $\mathrm{B}$ e $\mathrm{C}$ dos extratos socioeconômicos. Quanto à condição gestacional, 88,2\% estavam no segundo ou terceiro trimestre de gestação. Além disso, $49,5 \%$ apresentavam sobrepeso ou obesidade e $74,1 \%$ eram grávidas na condição de risco habitual (Tabela 1 ).

A Tabela 2 mostra uma análise descritiva (média e desvio padrão) das subescalas do BAQ e do escore total deste questionário. Também demonstra os resultados da comparação das médias por período gestacional. Destaca-se que tanto o escore total quanto as subescalas não se diferiram entre os grupos de primeiro, segundo e terceiro trimestres.

Tabela 1. Caracterização da amostra

\begin{tabular}{|c|c|c|}
\hline & $n$ & $\%$ \\
\hline \multicolumn{3}{|l|}{ Escolaridade } \\
\hline Ensino Fundamental & 81 & 21,0 \\
\hline Ensino Médio & 161 & 41,7 \\
\hline Ensino Superior/Pós-graduação & 144 & 37,3 \\
\hline \multicolumn{3}{|l|}{ Estado civil } \\
\hline Solteira & 86 & 22,3 \\
\hline Casada/Vive com o companheiro & 295 & 76,4 \\
\hline Divorciada/Viúva & 5 & 1,3 \\
\hline \multicolumn{3}{|l|}{ Número de filhos } \\
\hline 0 & 205 & 53,1 \\
\hline 1 & 91 & 23,6 \\
\hline 2 ou mais & 90 & 23,3 \\
\hline \multicolumn{3}{|l|}{ Nível socioeconômico } \\
\hline A & 18 & 4,7 \\
\hline B & 172 & 44,5 \\
\hline C & 166 & 43,1 \\
\hline DeE & 30 & 7,8 \\
\hline \multicolumn{3}{|l|}{ Estado nutricional } \\
\hline Baixo peso & 49 & 12,7 \\
\hline Adequado & 148 & 38,3 \\
\hline Sobrepeso & 100 & 25,9 \\
\hline Obesidade & 89 & 23,1 \\
\hline \multicolumn{3}{|l|}{ Período gestacional } \\
\hline Primeiro trimestre & 42 & 10,9 \\
\hline Segundo trimestre & 133 & 34,5 \\
\hline Terceiro trimestre & 211 & 54,6 \\
\hline \multicolumn{3}{|l|}{ Condição de risco } \\
\hline Alto risco & 97 & 25,1 \\
\hline Risco habitual & 289 & 74,9 \\
\hline
\end{tabular}


Tabela 2. Comparação de médias das subescalas do $B A Q$ e $B A Q$ total entre os períodos gestacionais

\begin{tabular}{|c|c|c|c|c|c|c|}
\hline \multirow{2}{*}{$B A Q$} & \multicolumn{3}{|c|}{ Período gestacional } & \multirow{2}{*}{ Amostra total } & \multirow{3}{*}{ Valor F } & \multirow{3}{*}{ Valor $p$} \\
\hline & Primeiro trimestre & Segundo trimestre & Terceiro trimestre & & & \\
\hline Subescalas & Média (DP) & Média (DP) & Média (DP) & Média (DP) & & \\
\hline Autodepreciação & $13,67(4,25)$ & $14,17(4,49)$ & $14,52(4,53)$ & $14,30(4,48)$ & 0,7 & 0,4 \\
\hline Gordura fotal & $38,28(11,16)$ & $37,62(10,34)$ & $36,47(10,19)$ & $37,06(10,35)$ & 0,8 & 0,4 \\
\hline Saliência do corpo & $21,09(4,63)$ & $20,86(4,61)$ & $20,70(3,84)$ & $20,80(4,19)$ & 0,1 & 0,8 \\
\hline Força e aptidão física & $17,71(3,40)$ & $17,52(3,11)$ & $18,32(3,11)$ & $17,98(3,16)$ & 2,7 & 0,06 \\
\hline BAQ Total & $122,07(18,26)$ & $121,22(17,17)$ & $121,35(17,26)$ & $121,38(17,30)$ & 0,04 & 0,9 \\
\hline
\end{tabular}

BAQ: Body Attitudes Questionnaire; BAQ total: escore total do Body Attitudes Questionnaire; DP: desvio padrão.

A partir da comparação das atitudes corporais quanto aos grupos por estado nutricional, os resultados foram significantes $(\mathrm{F}=36,32 ; \mathrm{p}=0,0001)$. $\mathrm{O}$ post hoc de Bonferroni identificou diferenças entre todos os grupos por estado nutricional para as médias do $\mathrm{BAQ}$, as quais aumentaram gradativamente entre os grupos de peso baixo $(108,28 \pm 12,57)$, adequado $(116,23 \pm 16,09)$, sobrepeso $(125,18 \pm 14,31)$ e obesidade $(132,9 \pm 16,41)$. Dessa forma, as gestantes com baixo peso tiveram uma imagem corporal mais positiva quando compradas àquelas com peso adequado $(\mathrm{p}=0,01)$, sobrepeso $(\mathrm{p}=0,0001)$ e obesidade $(\mathrm{p}=0,0001)$. Além disso, mulheres com peso adequado tinham reduzida preocupação relacionada ao corpo quando comparadas àquelas com sobrepeso $(\mathrm{p}=0,0001)$ e obesidade ( $\mathrm{p}=0,0001)$, e gestantes com sobrepeso em relação àquelas obesas $(\mathrm{p}=0,004)$.

Quanto à comparação por condição de risco, verificou-se que as gestantes classificadas com risco habitual apresentaram $(\mathrm{t}=-2,48 ; \mathrm{p}=0,013)$ média menor do BAQ $(120,01 \pm 17,10)$ do que aquelas com alto risco gestacional $(124,99 \pm 16,74)$.

A partir das associações entre as variáveis do estudo (dados completos não demonstrados), realizou-se uma análise de regressão linear múltipla forward, tendo os escores do BAQ como variável critério. Em primeiro lugar, o IMC foi inserido por apresentar maior valor de correlação $(r=0,339 ; p<0,05)$, seguido pelo nível socioeconômico $(r=-0,132 ; p<0,05)$. O IMC foi responsável por $11,3 \%$ da variância dos escores do BAQ $(\mathrm{F}=49,89$; $\mathrm{p}=0,0001)$. A variável nível socioeconômico não acrescentou explicação ao modelo e, por essa razão, foi excluída ao final da regressão.

\section{Discussão}

A partir dos achados encontrados, notou-se que os sentimentos de "atração física", "autodepreciação", "gordura total”, "saliência do corpo", "percepção de gordura nos membros inferiores do corpo" e "força e aptidão física" não foram diferentes entre os grupos por período gestacional. Dessa forma, as emoções e os pensamentos relacionados ao corpo de gestantes, possivelmente, foram similares entre todos os trimestres gestacionais. A análise das subescalas do BAQ em relação aos diferentes períodos gestacionais foi realizada anteriormente por pesquisadores internacionais ${ }^{2,15,17}$. Diferentemente dos achados da presente investigação, os estudos apontaram mudanças na imagem corporal ao longo da gestação.

Com relação à subescala "gordura total", estudos constataram que as gestantes se sentiam mais gordas antes da gestação do que em seu decorrer ${ }^{2,15,17}$. Além disso, essa preocupação foi maior no início da gravidez do que em momentos precedentes ao parto. Da mesma forma, para a subescala "saliência do corpo", Clark et al. ${ }^{2} \mathrm{e}$ Duncombe et al. ${ }^{15}$ relataram que as mulheres no período pré-gestacional achavam o peso e a forma corporais mais proeminentes do que no terceiro trimestre de gestação. Desse modo, a cobrança social do corpo magro aparentemente se dissipa ao longo dos períodos gestacionais, provocando um relaxamento da pressão durante essa fase para atingir o corporal ideal.

Embora as mulheres antes de engravidarem se sentissem mais gordas e com elevada saliência corporal, elas também se notavam mais atraentes, segundo a subescala "atração física" ${ }^{17}$. Diferentemente, Clark et al. ${ }^{2}$ e Duncombe et al. ${ }^{15}$ verificaram que a atratividade corporal se manteve constante, assim como no presente estudo. Para a mesma subescala, as mulheres no período pré-gestacional se sentiam mais fortes do que ao longo do desenvolvimento do feto $^{2,15,17}$. Portanto, conforme apontaram Meireles et al. ${ }^{11}$, os achados sobre imagem corporal em gestantes ainda permanecem incongruentes.

As subescalas "autodepreciação" e "percepção da gordura na porção inferior" não foram avaliadas nos estudos mencionados, não permitindo comparações. 
De acordo com a validação brasileira ${ }^{14}$, o BAQ contempla diversos aspectos das atitudes corporais globais das mulheres. Dessa forma, recomenda-se que o instrumento seja utilizado na sua totalidade.

Além disso, a partir dos resultados encontrados, verificou-se que a média de escore total do BAQ foi superior à pesquisa realizada nos Estados Unidos (média de 110,37 pontos; $\mathrm{DP}=14,37$ pontos) ${ }^{9}$. A amostra do presente estudo pode ter apresentado atitudes corporais mais negativas do que as gestantes americanas, muito embora os valores não tenham sido comparados estatisticamente. Vale ressaltar que o instrumento em questão não apresenta ponto de corte próprio, impossibilitando a classificação das gestantes quanto às suas atitudes corporais. Entretanto, ainda não há, no Brasil, questionário validado para esta população a fim de se verificar este componente da imagem corporal.

Por meio dos dados obstétricos, as gestantes foram classificadas quanto à condição de risco gestacional risco habitual e alto risco. A presente pesquisa avaliou as médias do BAQ com relação a esta classificação, sendo que gestantes de alto risco apresentaram crenças e sentimentos mais negativos relacionados ao corpo. A gestação classificada como alto risco, por si só, já merece maior atenção por parte dos profissionais que lidam diretamente com essa paciente, pois nesta condição a saúde física da mãe e/ou do feto pode estar mais comprometida ${ }^{4}$. Ademais, ressalta-se o aspecto da saúde psicológica, já que a condição de risco influenciou os sentimentos a respeito do próprio corpo de gestante nas mulheres avaliadas. Vale destacar que a avaliação do $\mathrm{BAQ}$, por grupo de condição de risco em gestantes brasileiras, é inédita na literatura e merece atenção dos pesquisadores.

Com base nos resultados da comparação do BAQ por estado nutricional, verificou-se que a preocupação com o próprio corpo das gestantes aumentou à medida que o IMC também aumentou. Esses dados são semelhantes não só com os achados da população em geral ${ }^{5}$, como também com estudos anteriormente realizados na população de gestantes ${ }^{8,20}$. No estudo de Sui et al. ${ }^{8}$, a insatisfação das grávidas com o peso e a forma corporal foi significativamente superior entre obesas do que aquelas com sobrepeso. De forma semelhante, Hauff e Demerath $^{20}$ observaram que gestantes com sobrepeso/ obesidade expressaram não apenas maiores preocupações com a aparência física quando comparadas àquelas com silhuetas mais magras, como também períodos mais curtos de amamentação.

Somado a estes achados, a regressão linear do presente estudo demonstrou que somente o IMC foi responsável pela variância dos escores do BAQ. Sui et al. ${ }^{8}$ também verificaram que o IMC foi preditor significante de imagem corporal negativa em gestantes australianas. Além disso, Kamysheva et al. ${ }^{16}$ constataram, pela mesma análise, que o IMC influenciou negativamente a atratividade das gestantes, ou seja, quanto maior o IMC delas, menores foram os sentimentos sobre a "atração física". Portanto, manter um IMC adequado associa-se a menores preocupações com o corpo, podendo influenciar positivamente na saúde materno-infantil ${ }^{21}$.

É importante enfatizar o ineditismo desta investigação no Brasil, bem como o seu tamanho amostral que supera o de pesquisas internacionais $2,6,7,15,16,18,22$. Contudo, não foi realizado um cálculo amostral que levasse em consideração as características obstétricas. Além disso, este estudo valeu-se do BAQ, o qual é validado para a população feminina adulta brasileira ${ }^{14}$, mas não tem todas as suas qualidades psicométricas testadas em grávidas. No entanto, a consistência interna desse questionário foi avaliada e considerada adequada para a amostra em questão $(\alpha=0,84)^{19}$. Foram identificados achados divergentes para as subescalas deste instrumento, que possivelmente se justificam em função das diferentes metodologias adotadas pelos estudos internacionais destacados ${ }^{2,15,17}$, os quais avaliaram as gestantes longitudinalmente.

Dessa forma, a utilização do corte transversal também pode ser considerada uma limitação, já que impossibilita a verificação dos efeitos de causalidade. Nesta pesquisa, a voluntária respondeu apenas uma única vez ao questionário, foi classificada pelo seu período gestacional, para então ser comparada às demais. Sendo assim, não é possível afirmar se as atitudes corporais de gestantes no primeiro trimestre se modificam ou se mantêm estáveis nos demais trimestres. Por outro lado, esta metodologia tem sido amplamente utilizada na avaliação da imagem corporal de gestantes ${ }^{7,23-25}$.

Concluiu-se que as atitudes corporais avaliadas em gestantes foram similares entre todos os trimestres gestacionais. Além disso, gestantes classificadas com alto risco e elevado IMC apresentaram imagem corporal mais negativa. Por fim, apenas o IMC foi preditor significativo de atitudes relacionadas à imagem corporal em grávidas. Sendo assim, a avaliação da imagem corporal nesta população é recomendada a fim de que um maior conhecimento sobre esse construto possa beneficiar essas mulheres, tendo em vista a sua saúde e o seu bem-estar psicológico.

\section{Agradecimentos}

À Coordenação de Aperfeiçoamento de Pessoal de Nível Superior (Capes) pela Bolsa de mestrado concedida à primeira autora. 
1. Watson B, Fuller-Tyszkiewicz M, Broadbent J, Skouteris $H$. The meaning of body image experiences during the perinatal period: a systematic review of the qualitative literature. Body Image. 2015; 14(3): 102-13.

2. Clark A, Skouteris H, Wertheim EH, Paxton SJ, Milgrom J. The relationship between depression and body dissatisfaction across pregnancy and the postpartum: a prospective study. J Health Psychol. 2009; 14(1):27-35.

3. Hodgkinson EL, Smith DM, Wittkowski A. Women's experiences of their pregnancy and postpartum body image: a systematic review and meta-synthesis. BMC Pregnancy Childbirth. 2014;14:330.

4. Brasil. Ministério da Saúde. Secretaria de Atenção à Saúde. Departamento de Ações Programáticas Estratégicas. Gestação de alto risco: manual técnico. Brasília (DF): Ministério da Saúde; 2012.

5. Ferreira ME, Castro MR, Morgado FF. Imagem corporal: reflexões, diretrizes e práticas de pesquisa. Juiz de Fora (MG): Editora UFJF; 2014.

6. Fuller-Tyszkiewicz M, Skouteris $H$, Watson B, Hill B. Body image during pregnancy: an evaluation of the suitability of the body attitudes questionnaire. BMC Pregnancy Childbirth. 2012;12(1):91.

7. Bagheri M, Dorosty A, Sadrzadeh-Yeganeh H, Eshraghian M, Amiri E, Khamoush-Cheshm N. Pre-pregnancy body size dissatisfaction and excessive gestational weight gain. Matern Child Health J. 2013; 17(4):699-707

8. Sui Z, Turnbull D, Dodd J. Effect of body image on gestational weight gain in overweight and obese women. Women Birth. 2013;26(4):267-72.

9. Sweeney AC, Fingerhut R. Examining relationships between body dissatisfaction, maladaptive perfectionism, and postpartum depression symptoms. J Obstet Gynecol Neonatal Nurs. 2013;42(5):551-61.

10. Silveira ML, Ertel KA, Dole N, Chasan-Taber L. The role of body image in prenatal and postpartum depression: a critical review of the literature. Arch Womens Ment Health. 2015; 18(3):409-21.

11. Meireles JF, Neves CM, Carvalho PH, Ferreira ME. Insatisfação corporal em gestantes: uma revisão integrativa da literatura. Cienc Saúde Coletiva. 2015;20(7):2089-101.

12. Thomas JR, Nelson JK, Silverman SJ. Métodos de pesquisa em atividade física. 5 ed. São Paulo: Artmed; 2012.

13. Associação Brasileira de Empresas de Pesquisa [Internet]. Critério de Classificação Econômica Brasil. 2012 [citado 2012 Dez 7]. Disponível em: http://www.abep.org/criterioBrasil.aspx
14. Scagliusi FB, Polacow VO, Cordás TA, Coelho D, Alvarenga M, Philippi ST, et al. Psychometric testing and applications of the Body Attitudes Questionnaire translated into Portuguese. Percept Mot Skills. 2005;101(1):25-41.

15. Duncombe D, Wertheim EH, Skouteris H, Paxton SJ, Kelly L. How well do women adapt to changes in their body size and shape across the course of pregnancy? J Health Psychol. 2008; 13(4):503-15.

16. Kamysheva E, Skouteris H, Wertheim EH, Paxton SJ, Milgrom J. Examination of a multi-factorial model of body-related experiences during pregnancy: the relationships among physical symptoms, sleep quality, depression, self-esteem, and negative body attitudes. Body Image. 2008;5(2):152-63.

17. Skouteris H, Carr R, Wertheim EH, Paxton SJ, Duncombe D. A prospective study of factors that lead to body image dissatisfaction during pregnancy. Body Image. 2005;2(4):347-61.

18. Hill B, Skouteris H, McCabe M, Fuller-Tyszkiewicz M. Body image and gestational weight gain: a prospective study. J Midwifery Womens Health. 2013;58(2): 189-94.

19. Streiner DL. Starting at the beginning: an introduction to coefficient alpha and internal consistency. J Pers Assess. 2003;80(1):99-103.

20. Hauff LE, Demerath EW. Body image concerns and reduced breastfeeding duration in primiparous overweight and obese women. Am J Hum Biol. 2012;24(3):339-49.

21. Silva JC, Amaral AR, Ferreira BS, Petry JF, Silva MR, Krelling PC. [Obesity during pregnancy: gestational complications and birth outcomes]. Rev Bras Ginecol Obstet. 2014;36(1 1):509-13. Portuguese.

22. McPhie S, Skouteris H, Fuller-Tyszkiewicz M, Hill B, Jacka F, O’Neil A. Relationships between mental health symptoms and body mass index in women with and without excessive weight gain during pregnancy. Midwifery. 2015;31(1):138-46.

23. Mills A, Schmied VA, Dahlen HG. 'Get alongside us', women's experiences of being overweight and pregnant in Sydney, Australia. Matern Child Nutr. 2013;9(3):309-21.

24. Sohn M, Bye E. Pregnancy and body image: analysis of clothing functions of maternity wear. Clothing Textiles Res J. 2015;33(1):64-78.

25. Radoš SN, Vraneš HS, Šunjić M. Limited role of body satisfaction and body image self-consciousness in sexual frequency and satisfaction in pregnant women. J Sex Res. 2014;51(5):532-41. 